\title{
Nudging travelers to favorable routes: The impact of visual communication and emotional responses on decision making
}

\author{
Stefan Fuest ${ }^{a}$ * , Monika Sester ${ }^{a}$, Amy L. Griffin ${ }^{b}$ \\ ${ }^{a}$ Institute of Cartography and Geoinformatics, Leibniz University Hannover, Germany, stefan.fuest@ikg.uni-hannover.de, \\ monika.sester@ikg.uni-hannover.de \\ ${ }^{b}$ School of Science, RMIT University Melbourne, Australia,amy.griffin@rmit.edu.au \\ * Corresponding author
}

Keywords: Map symbolization, visual variables, emotions, route choice, eco-consciousness, user study

\begin{abstract}
:
As urbanization increases, so does the demand for efficient forms of mobility. In particular, a high proportion of motorized individual transport can cause far-reaching problems for the transport system. Non-optimal distributions of traffic flows can overload the existing transport infrastructure, and lead to problems that may affect large parts of the urban population, such as traffic congestion at bottlenecks, or increased air pollution at critical locations. In both cases, a better distribution of traffic flows could contribute to improving the situation from a societal point of view.
\end{abstract}

In order to counteract the aforementioned developments, traffic authorities impose various measures, primarily focusing on direct intervention in the usable traffic infrastructure (e.g. speed limits, low emission zones or variable message signs). However, particularly in unfamiliar environments, the majority of route decisions are made prior to the start of the trip, based on route maps as provided by routing service applications or navigation systems. While the majority of existing services usually provide the most efficient route options for the individual traveler in terms of travel time, recent approaches try to guide the driver indirectly to a globally favorable route that temporarily improves the performance of the entire traffic system. Examples of this are collaborative routing approaches as developed by Graphmasters (2020) that aim to optimize traffic dynamics by reducing traffic jams and thus saving emissions. Similarly, a recently updated release of Google Maps intends to nudge travelers to eco-friendly route alternatives by default, instead of only suggesting time-efficient routes (Google, 2021).

While the described approaches could help improving the traffic situation from a system-optimal perspective, the resulting route recommendations may not necessarily match the individual preference of the traveler. If the recommended route clearly deviates from the individually most efficient route (e.g., in terms of travel time or distance), then this route option might be perceived as less reasonable to the traveler in terms of individual effort. Therefore, for effectively communicating the urgency of adapting route choice in favor of choosing a globally favorable route, route maps should attempt conveying the (negative) feeling that the traveler might get when being exposed to the situation.

Our idea to meet this problem is to use cartographic symbolization for communicating favorable, as well as nonfavorable route options to the traveler. Visual variables are an intuitive way for communicating spatial variations of map objects. Bertin's (1983) visual variable set has been elaborated over time - for example adding transparency or blurring / fuzziness (Kinkeldey et al., 2014). Like any other type of visual representation, map symbols can evoke different emotional responses in the viewer (Caquard \& Griffin, 2018). Therefore, it is important to investigate how emotions contribute to route choice decision making, as well as the extent to which they may play a role in achieving a behavior change. Previous research has shown that it is important to address people's emotions (Roeser, 2012), for them to understand the moral impact of the risks related to environmental phenomena. The challenge here is to use map symbols in a way that the symbolization adequately communicates the emotions that are expected to be associated with the communicated scenario. Providing intuitive visualizations that evoke anticipated emotions is expected to make the experience of the mapped phenomena more tangible to the map-reader.

The objective of this work is to investigate the potential of different types of cartographic symbolization for nudging a traveler's route choice towards a route that is favorable from a societal point of view. Additionally, we are interested in studying which types of emotions are evoked by viewing the different map representations. These emotional responses to map symbols are expected to affect the traveler's route choice decision.

We designed a user study for evaluating the impact of map symbolization and associated emotional responses on travelers' route choices. As part of the user study, we created a set of route maps that display two route options (one 
favorable and one non-favorable route) based on the current traffic situation or environmental conditions, while sharing the same start and end points. The route maps use different types of map modifications that are applied to line, area, or line + area geometries for symbolizing temporarily favorable and non-favorable parts of the environment.

Since we intend to simulate realistic route choice scenarios, all route maps are derived from real environments within major cities in Germany, taking care to avoid recognizable structures to minimize the impact of prior familiarity with the area. The structural characteristics of different real-world road networks may differ, and therefore could be considered factors that may influence route choice. An examination of the driving behavior literature identified several factors that should be controlled, including the number or right versus left turns, road size/speed, road network density, and travel direction (north to south vs south to north). We controlled for these factors to minimize their likelihood of influencing route choice. The major difference between the two provided route options is that one route is always slightly longer than the other. In the modified versions of the maps, this longer route is communicated as favorable (i.e., it either contributes to an improved traffic flow or better air quality in highly polluted areas). The shorter route, however, is not favored and therefore the visual modifications intend to nudge the traveler away from choosing this route - by making the route look less attractive and potentially evoking negative emotions.

The graphical differences in map symbolization are produced by the input data. The input data comprise traffic density measurements or the particulate matter concentration associated with a road segment. We defined a threshold value that separates the observed values into a range of favorable values and a range of non-favorable values. This threshold value can be based on fixed values that are defined by law (e.g., threshold for particulate matter concentrations in Germany), or defined dynamically based on the demand (e.g., average daily mean value). The ratio of the observed value to the threshold value is mapped to communicate route favorability.

Line object modifications include variations in line size and transparency (e.g., higher transparency represents a higher degree of pollution) or simplification or distortion of the geometry of line segments (Fuest et al., 2021). A further type of modification uses the traffic lights metaphor for symbolizing favorable and non-favorable parts of the routes - as frequently applied for real traffic maps. Modifications of areal objects include a blurring effect, color hue, as well as adding noise (scribble) to unfavorable parts of the map objects. Examples of combined modifications of line objects and areal objects within a single map representation include applying geometric distortion to line objects and using the visual variables color hue or blur for areal objects. Each modified map either uses one visual variable (line modification or areal modification), or two visual variables (combined) that symbolize the same phenomenon. For each of the modified maps, there is also a corresponding non-modified map depicting the same area, which does not show visual modifications based on the input data. All maps display the two route options and the surrounding road network.

The experiment uses a mixed design. A between-participants factor is the two scenarios: 1) traffic conditions, or 2) air quality. Within each of the two groups, participants are asked to complete the same set of three tasks. In the first task, participants are asked to make a route choice decision for each non-modified map, followed by all of the modified maps. For both sets of maps, the individual maps are shown in a randomized order. For the second task, participants are asked to provide free text descriptions justifying their route choice decision in the modified maps. This material is intended to be analyzed as part of a sentiment analysis (Meenar et al., 2019). For the third task of the experiment, we again show all modified maps one-by-one in a randomized order, and ask the participants to select the emotions they feel when viewing the favorable and non-favorable route in the maps - based on the Geneva emotion wheel (Scherer, 2005). The experiment concludes with a demographic questionnaire focusing on travel behavior, map usage and the participants' familiarity with commonly used map symbols in route maps.

The data analysis will focus on testing the following hypothesized relationships.

1) We expect a general shift towards choosing the longer, but favorable route, when comparing results for the non-modified and modified maps (Fuest et al., 2021).

2) We expect that combined modifications produce stronger emotional responses than line only or area only modifications, since they communicate favorability on different levels and that line modifications will be more effective for the traffic scenario while area modifications will be more effective for the air pollution scenario.

3) We expect a higher willingness for adapting route choice behavior (showing pro-social behavior), in the traffic condition scenario, rather than improving air pollution. We expect people to feel personally less affected by environmental impacts, and therefore also less responsible to act in a more altruistic way (Roeser, 2012).

4) In general, because loss framing is suggested to be generally more effective for evoking behavior change (Spence \& Pidgeon, 2010), we expect that communicating negative emotions for non-favorable routes is more effective for influencing route choice than communicating positive emotions for favorable routes. 
In conclusion, this work presents the design of a user study that investigates the influence of visual communication and emotions on route choice. We apply different types of cartographic symbolization for nudging a traveler's route choice towards a route that is favorable from a societal point of view. The results of the user study are expected to be useful for guiding map-makers in their design choices when creating route maps - by providing advice on intuitively symbolizing preferable, as well as non-preferable route options using visual variables.

\section{References}

Bertin, J. (1983). Semiology of graphics (W. J. Berg, Trans.). University of Wisconsin Press. (Original work published 1967).

Caquard, S., \& Griffin, A. (2018). Mapping emotional cartography. Cartographic Perspectives, (91), 4-16.

Fuest, S., Grüner, S., Vollrath, M., \& Sester, M. (2021). Evaluating the effectiveness of different cartographic design variants for influencing route choice, Cartography and Geographic Information Science, 48(2), 169-185.

Google (2021), accessed 2 June 2021, https://blog.google/products/maps/redefining-what-map-can-be-newinformation-and-ai/

Graphmasters (2020), accessed 2 June 2021, https://www.graphmasters.net/nunav

Kinkeldey, C., MacEachren, A. M., \& Schiewe, J. (2014). How to assess visual communication of uncertainty? A systematic review of geospatial uncertainty visualisation user studies. The Cartographic Journal, 51(4), 372-386.

Meenar, M., Flamm, B., \& Keenan, K. (2019). Mapping the emotional experience of travel to understand cycle-transit user behavior. Sustainability, 11(17), 4743.

Roeser, S. (2012). Risk communication, public engagement, and climate change: A role for emotions. Risk Analysis: An International Journal, 32(6), 1033-1040.

Scherer, K. R. (2005). What are emotions? And how can they be measured? Social science information, 44(4), 695-729.

Spence, A., \& Pidgeon, N. (2010). Framing and communicating climate change: The effects of distance and outcome frame manipulations. Global Environmental Change, 20(4), 656-667. 